\title{
El papel de Facebook Grupos en la educación médica peruana
}

\author{
The role of Facebook Groups in Peruvian medical education
}

\section{Sr. Editor:}

La red social Facebook, desde su lanzamiento el 2004, ha tenido un crecimiento exponencial llegando a formar parte de la vida personal, social y profesional de la población usuaria. Reportes publicados a mediados del 2018 por We Are Social en colaboración con Hootsuite, sitúan a esta red como la más utilizada con 2167 millones de usuarios en todo el mundo; de la misma manera en el Perú, el $69 \%$ de la población (22 millones de usuarios) cuenta con un perfil activo en esta interfaz (1).

La gratuidad y versatilidad que ofrece Facebook, ha permitido crear comunidades virtuales de usuarios con intereses comunes mediante grupos virtuales, donde intercambian información y promueven la libre discusión de los temas de interés colectivo. Este fenómeno también se ha extendido hacia comunidades estudiantiles y profesionales de la medicina $(2,3)$.

El uso educativo de las herramientas proporcionadas por Facebook grupos, debido a la accesibilidad y disponibilidad de la plataforma en cualquier lugar, momento y hora del día, viene generando gran expectativa en la colectividad médica (4). Un reciente meta-análisis, reportó que entre 45$96 \%$ de los estudiantes de medicina y médicos con edades comprendidas entre 18-25 años, hacen uso de las herramientas de Facebook durante al menos 30 minutos por día, convirtiéndose esta actividad parte de su rutina diaria $(3,5)$. Asimismo, uno de cada cuatro estudiantes de medicina utiliza esta red social con fines académicos mientras que el $50 \%$ restante, manifiesta que estaría dispuesto a hacerlo $(5,6)$.

La dinámica de Facebook grupos en la educación médica, contribuye a un mayor aprendizaje entre los médicos y estudiantes de medicina que lo utilizan $(7,8)$. En el Perú, debido a la elevada competitividad y exigencia que han generado el Examen Nacional de Medicina (ENAM) y el Examen Único Nacional de Residentado Médico Peruano (EXUN), surgieron en los últimos años Facebook grupos, que promueven la preparación médica continua, mediante la resolución de preguntas de opción múltiple, muchas de ellas recopiladas de los ENAM y EXUN de años anteriores, las cuales luego de ser publicadas son respondidas y discutidas por los usuarios de los grupos virtuales. Es importante mencionar que el Perú no es el único en el mundo donde se ha detectado este innovador medio de aprendizaje, México y España, también figuran como países de creación de grupos de similares características (tabla 1).

La red social Facebook tiene una elevada aceptación por parte de los estudiantes y profesionales de la medicina en todo el mundo, su uso con fines educativos está descrito en el modelo estructural de Facebook planteado por Mazmans y Usluels $(9,10)$, convirtiéndolo en un entorno efectivo de aprendizaje cuando se trata de la educación médica de pre y posgrado. Dada la gran expectativa que viene generando el uso de Facebook, nace la necesidad

1. Área de Medicina, Centro de Salud de Ambo, Huánuco, Perú.

2. Escuela Profesional de Medicina Humana, Universidad Nacional de San Cristóbal de Huamanga. Ayacucho, Perú.

3. Escuela Académico Profesional de Medicina, Universidad Nacional del Centro del Perú. Junín, Perú.

a. Médico Cirujano, MSc (c) en Gerencia de servicios en salud.

b. Estudiante de Medicina.

c. Administrador del grupo de Facebook "Desafio Ena(R)m 2019" 
Tabla 1. Principales grupos de Facebook que promueven la educación y preparación médica en México, España y Perú.

\begin{tabular}{|c|c|c|c|c|}
\hline $\begin{array}{l}\text { NOMBRE DEL GRUPO DE FACEBOOK } \\
\text { (CON URL) }\end{array}$ & $\begin{array}{l}\text { País de } \\
\text { creación }\end{array}$ & $\begin{array}{l}\text { Miembros a } \\
\text { enero } 2017\end{array}$ & $\begin{array}{l}\text { Miembros a } \\
\text { Agosto } 2018\end{array}$ & $\begin{array}{l}\text { Miembros a } \\
\text { marzo } 2019\end{array}$ \\
\hline $\begin{array}{l}\text { Repaso ENARM } \\
\text { https://www.facebook.com/groups/Repaso.ENARM/ }\end{array}$ & México & 53313 & 138751 & 149480 \\
\hline $\begin{array}{l}\text { Elrincondelamedicinainterna } \\
\text { https://www.facebook.com/groups/ } \\
\text { elrincondelamedicinainterna/ }\end{array}$ & España & 47895 & 87661 & 89154 \\
\hline $\begin{array}{l}\text { A Pasar El ENARM } \\
\text { https://www.facebook.com/ } \\
\text { groups/684024415088815/ }\end{array}$ & México & 22650 & 78643 & 83407 \\
\hline $\begin{array}{l}\text { Repaso RM } 2019 \mathrm{https}: / / \text { www.facebook.com/ } \\
\text { groups/383789398387940/?fref=ts }\end{array}$ & Perú & 22707 & 52779 & 56794 \\
\hline $\begin{array}{l}\text { Banco De Preguntas médicas https://www.facebook. } \\
\text { com/groups/BancoDePreguntasEnarm/ }\end{array}$ & México & 26222 & 29851 & 30199 \\
\hline $\begin{array}{l}\text { Desafío Ena(R)m } 2019 \text { https://www.facebook.com/gr } \\
\text { oups/1122258034475931/?fref=ts }\end{array}$ & Perú & 8282 & 19570 & 20205 \\
\hline $\begin{array}{l}\text { Recopilación ENARM https://www.facebook.com/ } \\
\text { groups/1461341477461834/ }\end{array}$ & México & 11137 & 13190 & 13450 \\
\hline $\begin{array}{l}\text { ¡Bancos Villamedic ... Discute tu clave! https://www. } \\
\text { facebook.com/groups/737431853087121/ }\end{array}$ & Perú & 914 & 5685 & 11430 \\
\hline $\begin{array}{l}\text { Repaso Médico - RM } 2018 \text { https://www.facebook. } \\
\text { com/groups/424589514375612/?ref=ts\&fref=ts }\end{array}$ & Perú & 3872 & 5680 & 5629 \\
\hline $\begin{array}{l}\text { Repaso ENAM } 2017 \\
\text { https://www.facebook.com/ } \\
\text { groups/1041706712558381/ }\end{array}$ & Perú & 809 & 2358 & 2642 \\
\hline $\begin{array}{l}\text { Repaso RM } 2017 \mathrm{https}: / / \text { www.facebook.com/groups/ } \\
881352595271081 / \text { ?ref=ts\&fref=ts }\end{array}$ & Perú & 1046 & 1764 & 1790 \\
\hline
\end{tabular}

urgente de investigaciones que estudien el impacto de Facebook grupos en el proceso de enseñanzaaprendizaje en la comunidad médico-estudiantil peruana.

\section{Wildor Samir Cubas ${ }^{1, a, c}$, Dercy Centeno-Leguía ${ }^{2, b, c}$, Angie Gómez-Briceño ${ }^{3, b, c}$.}

\section{Correspondencia:}

Wildor Samir Cubas Llalle

Área de Medicina del Centro de Salud de Ambo, Huánuco, Perú.

Jr. Aguilar 325, Huánuco, Perú

Correo electrónico: wsamircubas@gmail.com

Teléfono: 51947544610

\section{REFERENCIAS BIBLIOGRÁFICAS}

1. We Are Social. Global Digital Report 2018.New York: We Are Social; 2018. (Fecha de acceso 24 de marzo del 2019) Disponible en: https://digitalreport. wearesocial.com/

2. Chang LC, Guo JL, Lin HL. Cultural competence education for health professionals from pregraduation to licensure delivered using facebook: Twelve-month follow-up on a randomized control trial. Nurse Educ Today. 2017 ;59: 94-100. doi: 10.1016/j.nedt.2017.09.005

3. Pander T, Pinilla S, Dimitriadis K, Fischer MR. The use of Facebook in medical education-A literature review. GMS Z Med Ausbild. 2014;31(3):33. doi: 10.3205/zma000925

4. Nicolai L, Schmidbauer M, Gradel M, et al. Facebook Groups as a Powerful and Dynamic Tool in Medical 
Education: Mixed-Method Study. J Med Internet Res. 2017;19(12):e408. doi: 10.2196/jmir.7990

5. Guraya SY. The usage of social networking sites by medical students for educational purposes: a metaanalysis and systematic review. N Am J Med Sci. 2016; 8(7):268-78. doi: 10.4103/1947-2714.187131

6. Mejia CR, Flores SD, Verastegui-Díaz A, et al. Uso del Smartphone y de Facebook asociado a la autopercepción del rendimiento académico en estudiantes de Medicina peruanos. Rev cuba inf. cienc salud. 2017; 28(1):77-88.

7. Estus EL. Using Facebook within a geriatric pharmacotherapy course. Am J Pharm Educ. 2010; 74(8):145.

8. Gray K, Annabell L, Kennedy G. Medical students' use of Facebook to support learning: insights from four case studies. Med Teach. 2010; 32(12): 971-6. DOI: $10.3109 / 0142159 X .2010 .497826$
9. Cheston CC, Flickinger TE, Chisolm MS. Social media use in medical education: a systematic review. Acad Med. 2013;88(6):893-901. DOI: 10.1097/ACM.0b013e31828ffc23

10. Mazman SG, Usluel YK. Modeling educational usage of Facebook. Computers \& Education. 2010; 55(2):444-53. DOI: 10.1016/j.compedu.2010.02.008

Recibido: 25/03/2019 